\title{
COMMUNICATION
}

Cite this: DOI:

$10.1039 / \mathrm{x} 0 \times \mathrm{x} \times 0000 \mathrm{x}$

\section{A mechanochemical strategy for IRMOFs assembly based on pre-designed oxo-zinc precursors}

\author{
D. Prochowicz, ${ }^{a}$ K. Sokołowski, ${ }^{b}$ I. Justyniak, ${ }^{b}$ A. Kornowicz, ${ }^{a}$ D. Fairen-Jimenez, ${ }^{c}$ T. \\ Friščić $^{d}$ and J. Lewiński*a,b
}

Received 00th January 2012, Accepted ooth January 2012

DOI : $10.1039 / \times 0 \times \times 00000 x$

www.rsc.org/

We demonstrate a mechanochemical strategy that allowed the first successful mechanosynthesis of IRMOFs based on an oxo-centred SBU. The study indicates that controlling the acid-base relationships between reagents is key to mechanochemical synthesis of IRMOFs, and a pre-assembled oxo-zinc amidate cluster appeared as a very efficient precursor to IRMOFs.

Metal-organic frameworks (MOFs) have attracted major interest due to their applications in gas storage, separation, catalysis and sensing. ${ }^{1}$ One of the archetypal and still most prominent groups of MOFs are IRMOFs, based on $\left\{\mathrm{Zn}_{4} \mathrm{O}\right\}^{6+}$ oxo-zinc secondary building units (SBU) and organic carboxylate linkers. The most recognized member of this compound family is MOF-5, composed of oxozinc SBUs and terephtalate linkers. ${ }^{2}$ Conventional synthetic approaches to IRMOFs utilize either solvothermal or strongly basic conditions under which zinc salts undergo slow hydrolysis to form the oxo-zinc SBUs which further assemble with dicarboxylic acids into porous structures. ${ }^{3}$ Very recently, an alternative solvothermal strategy involving oxo-zinc acetate or benzoate clusters has been demonstrated for the MOF-5 synthesis. ${ }^{4}$

Over the last decade, chemical transformations driven by mechanical forces have recently experienced a renaissance of scientific interest. Mechanochemical synthesis, i.e. chemical synthesis performed by milling or grinding, has emerged as a powerful method for environmentally-friendly, clean, as well as energy-efficient construction of molecules and materials. ${ }^{5}$ Mechanosynthesis is advantageous to conventional solution-based or solvothermal synthetic methods as it allows shortening of reactions times while avoiding bulk solvents and high temperatures. So far, mechanosynthesis has successfully been utilized for the solid-state organic syntheses, ${ }^{6}$ the preparation of discrete metal complexes, ${ }^{7} 1 \mathrm{D}$ and $2 \mathrm{D}$ coordination polymers $^{8}$ and even porous MOFs ${ }^{9}$. The emerging solid-state approaches for MOF synthesis often utilize small amounts of a liquid (liquid-assisted grinding; LAG) or/and a salt (ion- and liquidassisted grinding; ILAG) ${ }^{9 \mathrm{~b}}$ which accelerate the synthesis and enable control over the MOF topology. Such approaches have been highly successful in providing rapid, room-temperature access to a number of microporous MOFs based on zinc, copper(II), and a handful of other metals, from a variety of simple precursors, ${ }^{\mathrm{a}-9 \mathrm{~d}, 9 \mathrm{~g}}$ including industrially attractive metal oxides. ${ }^{9 b, 9 e, 10}$ However, despite highly promising successes, mechanochemical methodologies have remained unable to generate the $\left\{\mathrm{Zn}_{4} \mathrm{O}\right\}^{6+} \mathrm{SBUs},{ }^{11}$ in that way leaving the assembly of archetypal IRMOFs out of reach of solventfree synthesis. Recently, we described a series of oxo-zinc carboxylate clusters with molecular geometries resembling the SBUs of IRMOF materials. ${ }^{12}$ Structural similarity sparked our interest in using such clusters as a means to access and explore IRMOFs using mechanochemistry.

We now describe the "SMART" (SBU-based Mechanochemical Approach for pRecursor Transformation) strategy that exploits the oxo-zinc clusters as pre-assembled precursors for mechanosynthesis of IRMOFs. In developing this strategy, we first explored a model oxo-zinc benzoate cluster $\left[\mathrm{Zn}_{4}\left(\mu_{4}-\mathrm{O}\right)\left(\mathrm{O}_{2} \mathrm{CPh}\right)_{6}\right]$ (1) as a mechanochemical precursor to IRMOFs, but then discovered that efficient mechanochemical reactivity requires switching to its amidate analogue $\left[\mathrm{Zn}_{4}\left(\mu_{4}-\mathrm{O}\right)(\mathrm{HNOCPh})_{6}\right](2)$. This study indicates that controlling the acid-base relationships between reagents is key to mechanochemical synthesis of MOF-5 as archetypal IRMOF. 


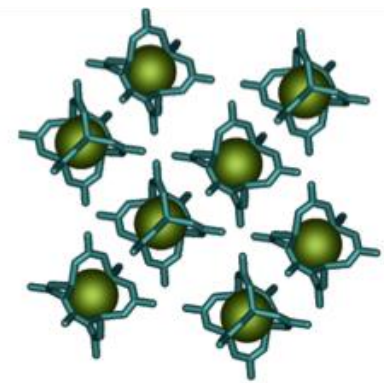

Zn-oxo precursor

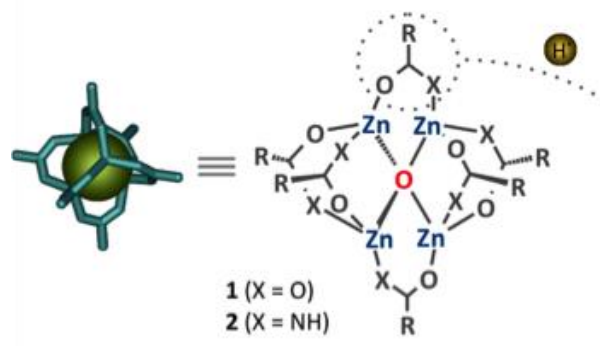

$2(X=N H)$

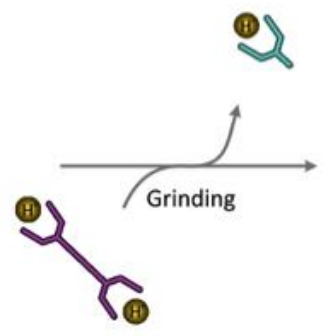

(1)

$\mathrm{p} K_{\mathrm{a}}=4.2$

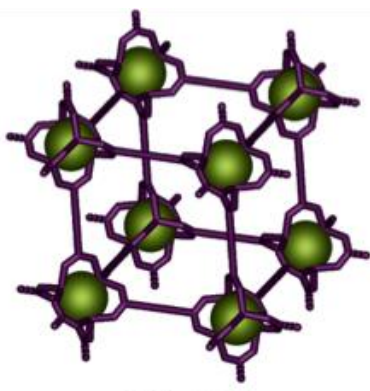

IRMOF lattice or<smiles>NC(=O)c1ccccc1</smiles>

$\mathrm{p} K_{\mathrm{a}}=14.5$

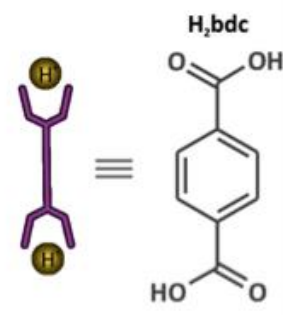

$\mathrm{p} K_{\mathrm{a} 1}=3.5$

$\mathrm{p} K_{22}=4.8$

Fig.1. A schematic representation of SMART strategy for the synthesis of MOF-5

Our initial attempts of MOF-5 mechanosynthesis focused on milling $\mathrm{ZnO}$ and terephthalic acid $\left(\mathrm{H}_{2} \mathrm{bdc}\right)$ in $4: 3$ stoichiometry, anticipating the in situ formation of the $\mathrm{Zn}_{4} \mathrm{O}$ SBU. However, powder X-ray diffraction (PXRD) patterns of milled reaction mixtures revealed only the formation of simple zinc terephthalates (see Fig. S2). Recognizing that the assembly of the $\mathrm{Zn}_{4} \mathrm{O}$ unit is a crucial obstacle to IRMOF mechanosynthesis, we therefore turned to pre-assembled oxo-zinc benzoate complex $\mathbf{1}$ as a potential mechanochemical precursor. ${ }^{12 \mathrm{~b}}$ Indeed, similar clusters have been utilized in solution chemistry for generation MOF-5 and analogues. ${ }^{4}$ However, after 60 min milling of 1 with $\mathrm{H}_{2}$ bdc in the required 1:3 stoichiometric ratio, PXRD revealed only broad Bragg's peaks consistent with the presence of $\mathrm{H}_{2}$ bdc, and no trace of MOF-5 (Fig. 2a-d).

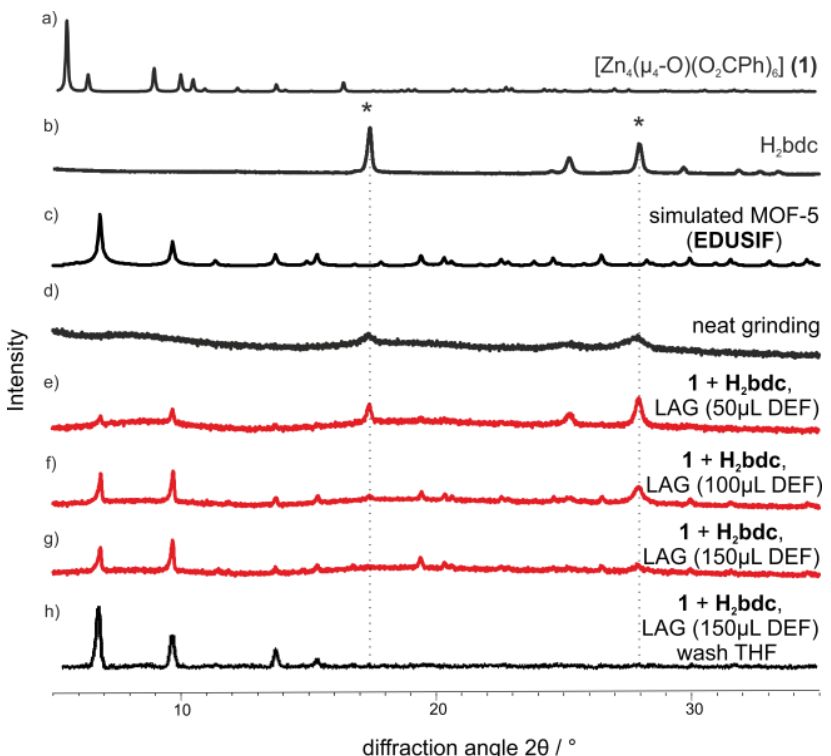

Fig. 2. Comparison of experimental and simulated PXRD patterns for the synthesis of MOF-5 by grinding 1 with $\mathrm{H}_{2}$ bdc: a) 1 . b) 1,4-benzenedicarboxylic acid. c) simulated pattern for MOF-5 (EDUSIF). d) neat grinding of $\mathbf{1}$ and $\mathrm{H}_{2}$ bdc. e-g) LAG of $\mathbf{1}$ and $\mathrm{H}_{2}$ bdc using DEF. h) after activating with THF. Reflection of $\mathrm{H}_{2}$ bdc are labeled with asterisks $(*)$.
Guided by the observation that small amounts of a liquid can enhance the scope and rate of mechanosynthesis as well as improve product crystallinity, we turned to liquid-assisted grinding (LAG). ${ }^{13}$ LAG of $\mathbf{1}$ and $\mathrm{H}_{2}$ bdc in the presence of $\mathrm{N}, \mathrm{N}$-diethylformamide (DEF; $50-150 \mu \mathrm{L}$ for $0.150 \mathrm{~g}$ of reagent mixture) led to the formation of MOF-5 after $60 \mathrm{~min}$, as indicated by PXRD analysis of the freshly milled reaction mixture (Fig. 2e-g). Although most of the reflections in the resulting PXRD pattern could be matched to MOF5 (Fig. 2c; CSD code EDUSIF), reflections of the residual $\mathrm{H}_{2}$ bdc were also apparent when using lower amounts of DEF. Increasing the amount of DEF in the reaction mixture improved the crystallinity of MOF-5 only slightly (Fig. 2e-g), suggesting that the principal problem in the mechanosynthesis of MOF-5 from $\mathbf{1}$ is poor reactivity of $\mathrm{H}_{2}$ bdc in the proposed proton-transfer reaction (Fig. 1). Bragg's peaks at $6.8^{\circ}$ and $9.7^{\circ}$ in the PXRD pattern of the as-synthesized mechanochemically sample of MOF-5 (designate as MOF- $5_{1}$ ) exhibit a different ratio of intensities than previously reported for MOF-5. These differences might be associated to residual zinc-based or benzoic acid species trapped in the MOF. ${ }^{14}$ This is supported by the observation that activating MOF- $5_{1}$ with anhydrous THF yields a material whose PXRD pattern is fully consistent with MOF-5 (Fig. 2h). ${ }^{15}$

Considering that acid-base reactivity is one of the driving forces for ligand exchange, we hypothesized that oxo-zinc clusters involving a more basic amidate ligand (generated from amides), rather than a carboxylate (generated from carboxylic acids), should favour the formation of IRMOFs in reactions with dicarboxylic acids. Importantly, oxo-zinc amidate ligands have previously never been explored in IRMOF synthesis. As the amidate source we first chose benzamide, exhibiting much less acidic character $(\mathrm{pKa}=14.5)$ than benzoic acid ( $\mathrm{pKa}=4.2$ ) (Fig. 1). The corresponding oxo-zinc amidate cluster $\left[\mathrm{Zn}_{4}\left(\mu_{4}-\mathrm{O}\right)(\mathrm{NHOCPh})_{6}\right](2)$ was obtained following a procedure previously reported for analogous oxo-zinc carboxylate clusters, ${ }^{12 \mathrm{~b}}$ by reacting three equivalents of benzamide with two equivalents of $\mathrm{Et}_{2} \mathrm{Zn}$ in $\mathrm{THF}$, followed by the addition of 0.5 equivalent of $\mathrm{H}_{2} \mathrm{O}$. Recrystallization from $\mathrm{THF}$ at $20^{\circ} \mathrm{C}$ gave crystals suitable for X-ray single-crystal determination, revealing 2 as a new example of this type of cluster family. ${ }^{16}$ The crystal structure of $\mathbf{2}$ consists of discrete clusters, based on the expected oxide-centered 
tetranuclear $\left[\mathrm{Zn}_{4} \mathrm{O}\right]^{6+}$ core surrounded by six $\mu$-1,2-amidate ligands. The $\mathrm{Zn}-\mathrm{O}_{\text {oxide }}$ distances fall into the expected range of 1.94-1.95 (Fig. S1).

Next, we applied 2 as the precursor for the mechanosynthesis of MOF- 5 by milling with three equivalents of $\mathrm{H}_{2}$ bdc. In contrast to the $1 / \mathrm{H}_{2}$ bdc system, neat grinding for $30 \mathrm{~min}$ led to the disappearance of all reflections characteristic of $\mathbf{2}$ and $\mathrm{H}_{2}$ bdc in the PXRD pattern and the appearance of broad reflections clearly consistent with MOF-5 and benzamide (Fig. 3e). Quantitative transformation of reactants was indicated by the absence of reactant absorption peaks in the Fourier-transform infrared (FTIR) spectrum of the ground product. After washing with THF to remove the benzamide byproduct, the resulting material (designated as MOF- $5_{2}$ ) exhibited a PXRD pattern fully consistent with MOF-5 (Fig. 3f). The PXRD analysis suggests that mechanosynthesis yields MOF-5 as poorly crystalline framework whose crystallinity, and therefore the sharpness of diffraction features, improves upon exposure to organic solvents. This view is consistent with SEM images of MOF-5 samples asprepared and after activating (see Fig. S10-S11). The importance of a mobile organic phase for crystallization of the MOF-5 structure is also evidenced by systematic LAG experiments involving increasing amounts of THF in the mechanochemical reaction of $\mathbf{2}$ and $\mathrm{H}_{2}$ bdc. Adding small volumes of THF improved the sharpness of MOF-5 reflections in the diffraction pattern (Fig. S3). Notably, the THF volume was between $25 \mu \mathrm{L}$ (ca. $0.3 \mathrm{mmol}, 0.75$ equivalents THF per zinc atom) and $100 \mu \mathrm{L}$ (ca. $1.2 \mathrm{mmol}, 3$ equivalents THF per zinc atom), which remains ca. 100 times below typical values used in solution-based synthesis. ${ }^{17}$ The described SMART strategy generates solid amide as the only byproduct, which can be re-utilized for the synthesis of pre-assembled oxo-zinc precursor. ${ }^{12 \mathrm{~b}}$

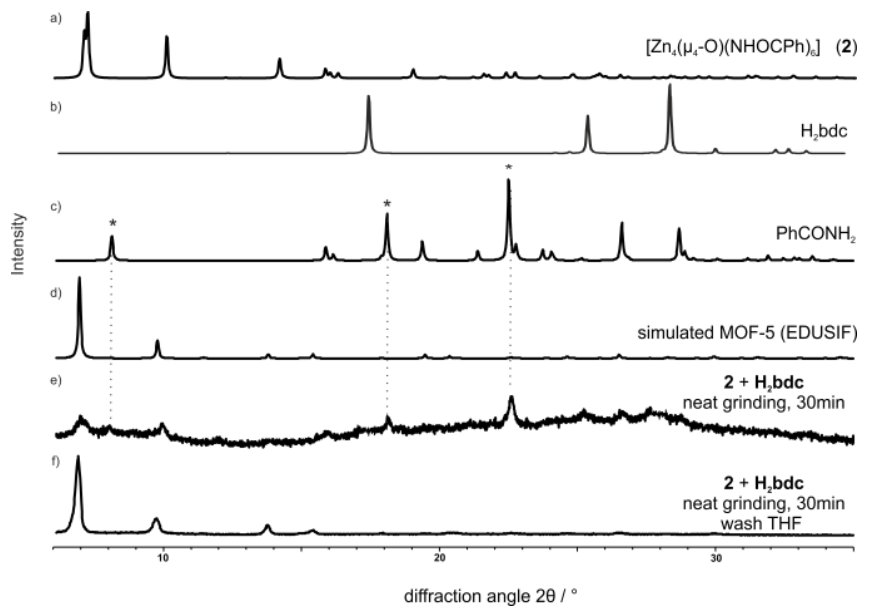

Fig. 3. Comparison of experimental and simulated PXRD patterns for the synthesis of MOF-5 by grinding 2 with $\mathrm{H}_{2}$ bdc: a) 2. b) 1,4-benzenedicarboxylic acid. c) benzamide. d) simulated pattern for MOF-5 (EDUSIF). e) neat grinding of 2 and $\mathrm{H}_{2}$ bdc. f) neat grinding of 2 and $\mathrm{H}_{2}$ bdc after activating with THF. Reflection of benzamide are labeled with asterisks $(*)$.

It is known that the surface area and gas adsorption capacity of MOFs are strongly affected by the method of their synthesis. ${ }^{18}$ Therefore, we decided to investigate thermal stability and gas adsorption properties of the mechanochemically synthesized materials. Samples of MOF- $5_{1}$ and MOF- $5_{2}$ were all activated by washing with anhydrous THF and desolvation under a dynamic vacuum $\left(10^{-6} \mathrm{mbar}\right)$ at $100^{\circ} \mathrm{C}$ for $12 \mathrm{~h}$, yielding samples MOF- $5_{1 \mathrm{a}}$ and MOF- $5_{2 \mathrm{a}}$ that have no guest within frameworks as confirmed by ${ }^{1} \mathrm{H}$ NMR and thermogravimetric (TGA) analysis. ${ }^{19}$ At $77 \mathrm{~K}, \mathrm{MOF}-$ $5_{1 \mathrm{a}}$ and MOF- $5_{2 \mathrm{a}}$ exhibit type $\mathrm{I} \mathrm{N}_{2}$ adsorption isotherms typical for microporous materials, with maximum uptakes of $450 \mathrm{~cm}^{3} \mathrm{~g}^{-1}$ and
$650 \mathrm{~cm}^{3} \mathrm{~g}^{-1}$ (at standard temperature and pressure (STP); Fig. S12), respectively. The Brunauer-Emmett-Teller (BET) areas for MOF-5 and MOF- $5_{2 \mathrm{a}}$, calculated from $\mathrm{N}_{2}$ adsorption isotherms are $1831 \mathrm{~m}^{2}$ $\mathrm{g}^{-1}$ and $2345 \mathrm{~m}^{2} \mathrm{~g}^{-1}$, respectively. We compared our $\mathrm{N}_{2}$ adsorption results with molecular simulation using grand canonical Monte Carlo (GCMC). Fig.4a and Fig.S15 show the comparison between experimental and GCMC isotherms. The BET area of the simulated isotherm is $3134 \mathrm{~m}^{2} / \mathrm{g}$, which corresponds to a scaling factor of $\varphi=$ $0.739 .^{20}$ As can be seen in the inset of Fig. 4a, both the experimental and simulated isotherms show the characteristic step of MOF-5 at very low pressure (ca. $0.002 \mathrm{P} / \mathrm{P} 0$ ) and the unusual sigmoidal shape of the curves. ${ }^{21}$

By representing snapshots and density distributions of the $\mathrm{N}_{2}$ isotherms, GCMC simulation shows that the step is related to the existence of adsorption sites with different interaction strength. In this way, $\mathrm{N}_{2}$ molecules are first adsorbed close to the metal cluster before being adsorbed in the rest of the porosity (Fig.4b and Fig. S16).
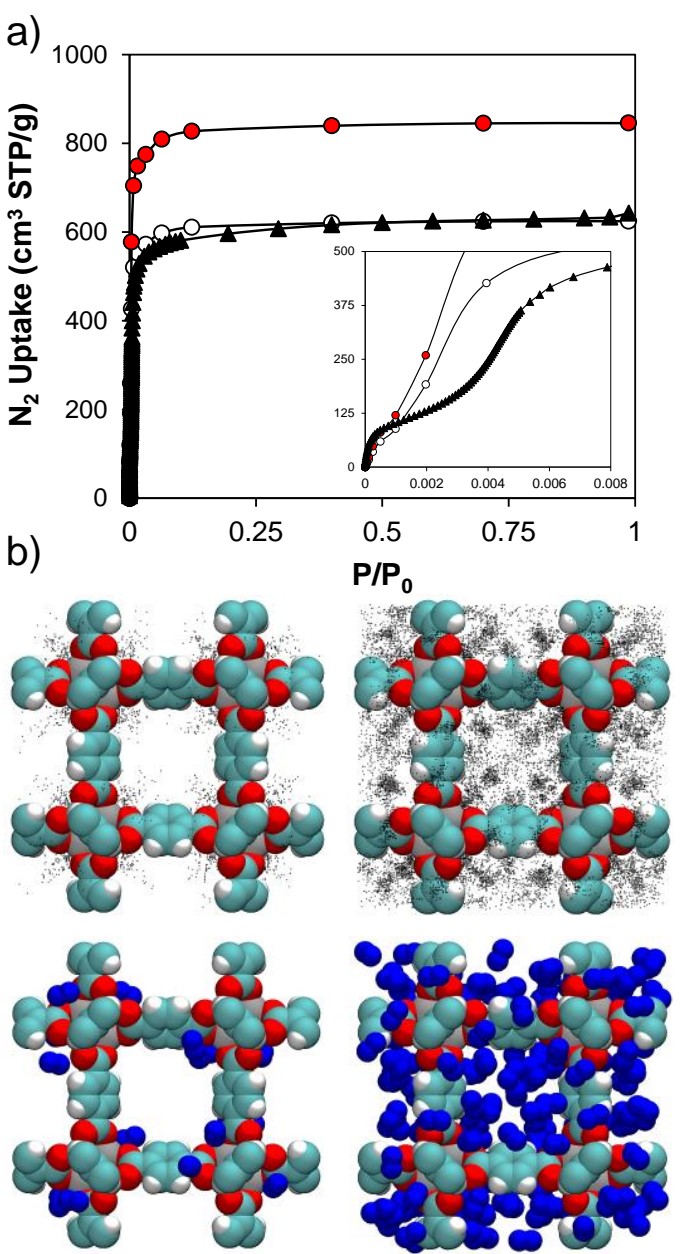

Fig. 4. a) Adsorption isotherms of $\mathrm{N}_{2}$ on MOF-5 at $77 \mathrm{~K}$. Experiment MOF-5 $5_{2 \mathrm{a}}$, black triangles; GCMC, red circles; scaled GCMC $(\phi=0.739)$. b) Density distributions (top) and snapshots (bottom) of $\mathrm{N}_{2}$ adsorption on MOF-5 at $77 \mathrm{~K}$ at low (left) and high (right) loadings, obtained by GCMC. Black points and blue spheres represent the $\mathrm{N}_{2}$ molecules in the density distributions and snapshots, respectively.

We showed previously that the presence of unusual sigmoidal behavior during the adsorption process is a result of relatively weak guest-host interactions, which is in turn a consequence of the relatively open nature of the MOF framework compared with other microporous adsorbents. ${ }^{21 \mathrm{~b}}$ These values are moderate, compared with MOF-5 materials synthesized by different solvothermal 
methods, ${ }^{22}$ but significantly higher than those obtained for MOF-5 from room-temperature precipitation $\left(\mathrm{S}_{\mathrm{BET}}=700 \mathrm{~m}^{2} \mathrm{~g}^{-1}\right) \cdot{ }^{23}$ Evaluation of the $\mathrm{H}_{2}$ adsorption in MOF-5 $5_{1 \mathrm{a}}$ and MOF-5 $5_{2 \mathrm{a}}$ at $77 \mathrm{~K}$ revealed an uptake of $0.82 \mathrm{wt} \%$ and $1.3 \mathrm{wt} \%\left(92 \mathrm{~cm}^{3} \mathrm{~g}^{-1}\right.$ and $150 \mathrm{~cm}^{3} \mathrm{~g}^{-1}$ STP, respectively; Fig. S13-S14). These values are similar to those originally reported for MOF-5 $(1.3 \mathrm{wt} \%) .^{2 \mathrm{~b}}$

In summary, we have demonstrated the first mechanochemical strategy for the synthesis metal-organic frameworks based on oxocentred SBUs, specifically the member of the archetypal IRMOF materials, central to the history and development of modern MOF chemistry. In contrast to solution-based ligand exchange reactions, the efficient mechanosynthesis of IRMOFs mandates the use of a basic pre-assembled oxo-zinc amidate precursor. We believe that the presented results open new possibilities in the clean and efficient synthesis of microporous MOFs, as well as add a new strategy for exploring the phase space of new metal-organic frameworks.

The authors would like to acknowledge the project operated within the Foundation for Polish Science Team Programme cofinanced by the EU "European Regional Development Fund" TEAM/2011-7/8, and the European Union in the framework of Regional Development Fund through the Joint UW and WUT International PhD Program of Foundation for Polish Science "Towards Advanced Functional Materials and Novel Devices" (MPD/2010/4) (D.P.) for financial support. We thank I. Dzięcielewski for performing SEM analyses

\section{Notes and references}

a Department of Chemistry, Warsaw University of Technology, Noakowskiego 3, 00-664 Warsaw (Poland)

${ }^{b}$ Institute of Physical Chemistry, Polish Academy of Sciences, Kasprzaka 44/52, 01-224 Warsaw (Poland)

${ }^{c}$ Department of Chemical Engineering \& Biotechnology, University of Cambridge, Pembroke Street, Cambridge CB2 3RA (UK)

${ }^{d}$ Department of Chemistry and FRQNT Centre for Green Chemistry and Catalysis, McGill University, 801 Sherbrooke St. W., Montréal, H3A0B8, (Canada)

$\dagger$ Electronic Supplementary Information (ESI) available: All experimental details and characterization data. See DOI: $10.1039 / \mathrm{c} 000000 \mathrm{x} /$

1 (a) K. Sumida, D. L. Rogow, J. A. Mason, T. M. McDonald, E. D. Bloch, Z. R. Herm, T.-H. Bae and J. R. Long, Chem. Rev., 2012, 112 , 724; (b) M. P. Suh, H. J. Park, T. K. Prasad and D.-W. Lim, Chem. Rev., 2012, 112, 782; (c) M. Schröder, Top. Curr. Chem., 2010, 293, 1; (d) J. Lee, O. K. Farha, J. Roberts, K. A. Scheidt, S. T. Nguyen, J. T. Hupp, Chem. Soc. Rev., 2009, 38, 1450.

2 (a) M. Eddaoudi, D. B. Moler, H. Li, B. Chen, T. M. Reineke, M. O'Keeffe and O. M. Yaghi, Acc. Chem. Res., 2001, 34, 319; (b) M. Eddaoudi, J. Kim, N. Rosi, D. Vodak, J. Wachter, M. O'Keeffe and O. M. Yaghi, Science 2002, 295, 469; (c) D. J. Tranchemontagne, J. L. Mendoza-Cortés, M. O'Keeffe and O. M. Yaghi, Chem. Soc. Rev., 2009, 38, 1257.

3 S. R. Caskey and A. J. Matzger, Material Matters, 2009, 4, 111.

4 S. Hausdorf, F. Baitalow, T. Boehle, D. Rafaja and F. O. R. L. Mertens, J. Am. Chem. Soc., 2010, 132, 10978.

5 (a) S. L. James, C. J. Adams, C. Bolm, D. Braga, P. Collier, T. Friščić, F. Grepioni, K. D. M. Harris, G. Hyett, W. Jones, A. Krebs, J. Mack, L. Maini, A. G. Orpen, I. P. Parkin, W. C. Shearouse, J. W. Steed and D. C. Waddell Chem. Soc. Rev., 2012, 41, 413; (b) T. Friščić, Chem. Soc. Rev., 2012, 41, 3493; (c) D. Braga, S. L. Giaffreda, F. Grepioni, A. Pettersen, L. Maini, M. Curzi and M. Polito, Dalton Trans., 2006, 1249.

6 G. Kaupp, Top. Curr. Chem., 2005, 254, 95.

7 (a) A. Lazuen Garay, A. Pichon and S. L. James, Chem. Soc. Rev., 2007, 36, 846; (b) J. Lewiński, M. Dutkiewicz, M. Lesiuk, W.
Śliwiński, K. Zelga, I. Justyniak and J. Lipkowski, Angew. Chem. Int. Ed., 2010, 49, 8266; (c) T. Friščić, E. Meštrović, D. Škalec-Šamec, B. Kaitner and L. Fábián, Chem. Eur. J., 2009, 15, 12644.

8 (a) D. Braga, M. Curzi, A. Johansson, M. Polito, K. Rubini and F. Grepioni, Angew. Chem. Int. Ed., 2006, 45, 142; (b) W. J. Belcher, C. A. Longstaff, M. R. Neckenig and J. W. Steed, Chem. Commun., 2002, 1602; (c) W. B. Yuan, T. Friščić, D. Apperley and S. L. James, Angew. Chem. Int. Ed., 2010, 49, 3916; (d) D. Prochowicz, I. Justyniak, A. Kornowicz, T. Kaczorowski, Z. Kaszkur and J. Lewiński, Chem. Eur. J., 2012, 18, 7367.

9 (a) A. Pichon, A. Lazuen-Garay and S. L. James, CrystEngComm, 2006, 8, 211; (b) T. Friščić, I. Halasz, P. J. Beldon, A. M. Belenguer, F. Adams, S. A. J. Kimber, V. Honkimäki and R. E. Dinnebier, Nature Chem., 2013, 5, 66; (c) W. Yuan, A. Lazuen Garay, A. Pichon, R. Clowes, C. D. Wood, A. I. Cooper and S. L. James, CrystEngComm, 2010, 12, 4063; (d) H. Sakamoto, R. Matsuda and S. Kitagawa, Dalton Trans., 2012, 41, 3956; (e) P. Wang, G. Li, Y. Chen, S. Chen, S. L. James and W. Yuan, CrystEngComm, 2012, 14, 1994; (f) N. K. Singh, M. Hardi and V. P. Balema, Chem. Commun., 2013, 49, 972; (g) M. Klimakow, P. Klobes, A. F. Thunemann, K. Rademann and F. Emmerling, Chem. Mater., 2010, 22, 5216.

10 A. Czaja, E. Leung, N. Trukhan, U. Muller in Metal-Organic Frameworks: Applications from Catalysis to Gas Storage, 340 (Wiley-VCH 2011).

11 For very rare example of the application of pre-assembly SBUs for the synthesis of certain MOFs in solution see: Ref. 4 and (a) C. Serre, F. Millange, S. Surblé and G. Férey, Angew. Chem. Int. Ed., 2004, 43, 6286; (b) V. Guillerm, S. Gross, C. Serre, T. Devic, M. Bauer and G. Férey, Chem. Commun., 2010, 46, 767.

12 (a) J. Lewiński, W. Bury, M. Dutkiewicz, M. Maurin, I. Justyniak and J. Lipkowski, Angew. Chem. Int. Ed., 2008, 47, 573; (b) W. Bury, I. Justyniak, D. Prochowicz, A. Rola-Noworyta and J. Lewiński, Inorg. Chem., 2012, 51, 7410; (c) W. Bury, I. Justyniak, D. Prochowicz, Z. Wróbel and J. Lewiński, Chem. Commun., 2012, 48, 7362 .

13 (a) G. A. Bowmaker, Chem. Commun., 2013, 49, 334; (b) T. Friščić and W. Jones, Cryst. Growth Des., 2009, 9, 1621; (c) K. L. Nguyen, T. Friščić, G. M. Day and L. F. Gladden, Nature Mater., 2007, 6, 206.

14 J. Hafizovic, M. Bjorgen, U. Olsbye, P. D. C. Dietzel, S. Bordiga, C. Prestipino, C. Lamberti and K. P. Lillerud, J. Am. Chem. Soc., 2007, 129, 3612.

15 The yield after desolvation was found to be $72 \%$. The reaction was performed at $0.1 \mathrm{mmol}$ scale, by placing a mixture of $\mathbf{1}(0.1 \mathrm{~g} ; 0.1$ mmol $)$ and $\mathrm{H}_{2}$ bdc $(0.049 \mathrm{~g} ; 0.3 \mathrm{mmol})$ in the presence of $150 \mu \mathrm{L}(1.35$ mmol, ca. 3.4 equivalents per zinc atom) of DEF into a $10 \mathrm{ml}$ stainless steel jar with two $7 \mathrm{~mm}$ diameter stainless steel balls. The mixture was ground for $60 \mathrm{~min}$ in a Retsch MM200 mill at $30 \mathrm{~Hz}$.

16 R. P. Davies, D. J. Linton, P. Schooler, R. Snaith and A. E. H. Wheatley, Chem. Eur. J., 2001, 7, 3696.

17 T. Friščić, S. L. Childs, S. A. A. Rizvi and W. Jones, CrystEngComm, 2009, 11, 418.

18 N. Stock and S. Biswas, Chem. Rev., 2012, 112, 933.

19 TGA studies revealed that MOF-5 $5_{1 \mathrm{a}}$ and MOF-5 $5_{2 \mathrm{a}}$ are stable up to approximately $400^{\circ} \mathrm{C}$; see Fig. S8-S9.

20 D. Fairen-Jimenez, R. Galvelis, A. Torrisi, A. D. Gellan, M. T. Wharmby, P. A. Wright, C. Mellot-Draznieks and T. Düren, Dalton Trans., 2012, 41, 10752.

21 (a) J. P. Marco-Lozar, J. Juan-Juan, F. Suárez-García, D. CazorlaAmorós and A. Linares-Solano, Int J Hydr Energy, 2012, 37, 2370; (b) D. Fairen-Jimenez, N. A. Seaton and T. Düren, Langmuir, 2010, 26, 14694.

22 (a) H. Li, M. Eddaoudi, M. O'Keeffe and O. M. Yaghi, Nature, 1999, 402, 276; (b) J. Rowsell, A. Millward, K. Park and O.M. Yaghi, J. Am. Chem. Soc., 2004, 126, 5666; (c) B. Panella, M. Hirscher, H. Putter and U. Muller, Adv. Funct. Mater., 2006, 16, 520; (d) H. Kim, S. Das, M. G. Kim, D. N. Dybtsev, Y. Kim and K. Kim, Inorg. Chem., 2011, 50, 3691; (e) M. Ma, D. Zacher, X. Zhang, R. A. Fischer and N. Metzler-Nolte, Cryst. Growth Des., 2011, 11, 185.

23 L. Huang, H. Wang, J. Chen, Z. Wang, J. Sun, D. Zhao and Y. Yan, Microporous Mesoporous Mater., 2003, 58, 105. 